PAPER: DISORDERED SYSTEMS, CLASSICAL AND QUANTUM

\title{
Out-of-equilibrium 2D Ising spin glass: almost, but not quite, a free-field theory
}

\section{Recent citations}

To cite this article: L A Fernandez et al J. Stat. Mech. (2018) 103301

View the article online for updates and enhancements.

\section{IOP ebooks}

Bringing you innovative digital publishing with leading voices

to create your essential collection of books in STEM research.

Start exploring the collection - download the first chapter of every title for free. 


\section{Out-of-equilibrium 2D Ising spin glass: almost, but not quite, a free-field theory}

L A Fernandez
G Parisi ${ }^{3,4,5}$ and J E Marinari
J

1 Departamento de Física Teórica, Facultad de Ciencias Físicas, Universidad Complutense de Madrid, Madrid 28040, Spain

${ }^{2}$ Instituto de Biocomputación y Física de Sistemas Complejos (BIFI), 50018 Zaragoza, Spain

3 Dipartimento di Fisica, Sapienza Università di Roma, I-00185 Rome, Italy

${ }^{4}$ Nanotec, Consiglio Nazionale delle Ricerche, I-00185 Rome, Italy

${ }^{5}$ Istituto Nazionale di Fisica Nucleare, Sezione di Roma 1, I-00185 Rome, Italy

${ }^{6}$ Departamento de Física and Instituto de Computación Científica Avanzada (ICCAEx), Universidad de Extremadura, 06071 Badajoz, Spain

E-mail: ruiz@unex.es

Received 24 May 2018

Accepted for publication 19 September 2018

Published 23 October 2018

Online at stacks.iop.org/JSTAT/2018/103301

https://doi.org/10.1088/1742-5468/aae2e1

Abstract. We consider the spatial correlation function of two-dimensional Ising spin glass under out-of-equilibrium conditions. We pay special attention to the scaling limit reached upon approaching zero temperature. The fieldtheory of a non-interacting field makes a surprisingly good job of describing the spatial shape of the correlation function of the out-of-equilibrium EdwardsAnderson Ising model in two dimensions.

Keywords: spin glasses 


\section{Contents}

1. Introduction 2

2. Model and observables 3

3. On the spatial structure of the correlations 5

4. Equilibrium Edwards-Anderson correlations and the theory of a free field

5. Out-of-equilibrium Edwards-Anderson correlations and the $\begin{array}{ll}\text { theory of a free field } & 11\end{array}$

6. Conclusions $\quad 14$

Acknowledgments ...................................................................... 14

Appendix. The out-of-equilibrium dynamics of the free scalar field ... 14

A.1. Integral estimators of dynamic correlations ........................................... 15

A.2. Asymptotic behavior of $F_{D}(y, w)$ (large $y$ at fixed $w$ ) ............................... 17

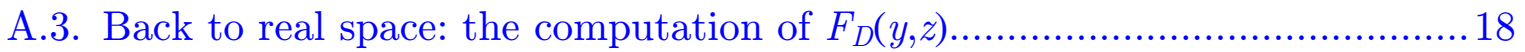

$\begin{array}{ll}\text { References } & 19\end{array}$

\section{Introduction}

The importance of characterizing the spatial range of spin-glass correlations has been long recognized, both under equilibrium [1, 2] and out-of-equilibrium conditions [3-22]. These correlations may be characterized though the overlap-overlap correlation function (for definitions, see section 2). However, we still lack analytical control over the spatial shape of this correlation function, which is a great nuisance for numerical work.

Here, we study the overlap-overlap correlation function for Ising spin glass in the spatial dimension $D=2$ both as a function of time and of spatial separation. Our numerical analysis is performed on lattices large enough to be representative of infinite system-size limit. The two dimensional (2D) Ising spin glass undergoes a $T=0$ phase transition; however, we hope that our results would apply equally in Ising spin glasses above the lower critical dimension (which is believed to be $D \simeq 2.5$ [23]) in the paramagnetic phase. In fact, recent experiments on a film geometry [17, 21, 24-26] motivated us to undertake a large-scale numerical simulation of the out-of-equilibrium dynamics of the $D=2$ spin glass [22]. These systems will occasionally behave as if living in a spin glass phase, but most of the time they will cross over to the dynamical critical behavior of the 2D Ising spin glass; i.e. paramagnetic phase behavior. Our aim here is to present a more field-theoretically minded analysis of the correlation function, as compared with our previous phenomenological analysis [22].

It came to us as a real surprise that the Langevin dynamics for the free scalar field make an excellent job of describing the spatial dependence of spin-glass correlations. 
Of course, at least in an equilibrium setting [27, 28], large-distance correlations in a paramagnetic phase (and the $D=2$ Ising spin glass has only a paramagnetic phase for $T>0$ ) should be given by free-field theory. What is a surprise is that free-field theory is very accurate also at short distances. Furthermore, in the large-time limit of an equilibrated system, free-field theory can be made virtually exact for the spin glass through a logarithmic wave-function renormalization (because of the vanishing anomalous dimension [29]). In fact, we are able to parameterize in a very simple way the rather heavy corrections to scaling found in a previous equilibrium study [30].

The remaining part of this work is organized as follows. In section 2 we shall describe the model and the basic spin-glass correlation function that we compute (for further technical details, see [22]). In section 3 we elaborate on the implications of scale invariance for the spatial shape of the correlation function. The relationship between the spinglass correlations and the free-field propagator is considered in equilibrium (section 4) and out of equilibrium (section 5). Our conclusions are presented in section 6. A number of results regarding the free-field propagator are derived and discussed in the appendix.

\section{Model and observables}

Our dynamic variables are Ising spins, $s_{\boldsymbol{x}}= \pm 1$, placed in the nodes of a square lattice of linear dimension $L$. Their interaction is given by the Edwards-Anderson Hamiltonian [31, 32] with nearest-neighbor couplings and periodic boundary conditions

$$
\mathcal{H}=-\sum_{\langle\boldsymbol{x}, \boldsymbol{y}\rangle} J_{\boldsymbol{x}, \boldsymbol{y}} s_{\boldsymbol{x}} s_{\boldsymbol{y}}
$$

We consider quenched disorder [33], which means that the couplings $J_{\boldsymbol{x}, \boldsymbol{y}}$ are fixed once for all. The couplings are drawn from the bimodal probability distribution $\left(J_{\boldsymbol{x}, \boldsymbol{y}}= \pm 1\right.$ with equal probability). Every set $\left\{J_{\boldsymbol{x}, \boldsymbol{y}}\right\}$ defines a sample. We have simulated $L=512$, which is large enough to be insensitive to the finite size effects (see section 4). Notice that the $T=0$ phase transition is universal (i.e. it is independent of the type of disorder, see for example [29]).

Our numerical protocol is as follows. We start from a fully disordered spin configuration (representative of infinite temperature), which is instantaneously placed at the working temperature $T$ at the initial time $t_{\mathrm{w}}=0$. Standard Metropolis dynamics at fixed $T$ follow. Our time unit is a full-lattice sweep, which roughly corresponds to one picosecond [34]. We have simulated a multi-spin code of an $L=512$ lattice for a wide range of temperatures $(0.5 \leqslant T \leqslant 1.1)$. The number of simulated samples was 96 . For each sample, we ran 256 replicas (for $T \geqslant 0.55$ ) or 264 replicas (for $T=0.5$ ).

The overlap correlation function (see [16] for a detailed discussion) is computed from the replica field

$$
q^{\alpha, \beta}\left(\boldsymbol{x}, t_{\mathrm{w}}\right)=s^{(\alpha)}\left(\boldsymbol{x}, t_{\mathrm{w}}\right) s^{(\beta)}\left(\boldsymbol{x}, t_{\mathrm{w}}\right), \quad(\alpha \neq \beta) .
$$

The $\left\{s^{(\alpha)}\left(\boldsymbol{x}, t_{\mathrm{w}}\right)\right\}$ are real replicas ( $\alpha$ is the so-called replica index): replicas with different replica indices evolve under the same set of couplings $\left\{J_{\boldsymbol{x}, \boldsymbol{y}}\right\}$ but are otherwise statistically independent. Hence, our correlation function is 


$$
C_{4}\left(\boldsymbol{r}, t_{\mathrm{w}} ; T\right)=\overline{\left\langle q^{\alpha, \beta}\left(\boldsymbol{x}, t_{\mathrm{w}}\right) q^{\alpha, \beta}\left(\boldsymbol{x}+\boldsymbol{r}, t_{\mathrm{w}}\right)\right\rangle},
$$

where one first takes the average over the thermal noise and the initial conditions, denoted by $\langle\ldots\rangle$. The average over the random couplings, denoted by an overline, is only computed afterwards. We shall restrict ourselves to displacement vectors along one of the lattice axes (the choice between $\boldsymbol{r}=(r, 0)$ or $\boldsymbol{r}=(0, r)$ is immaterial, so we average over the two), and use the shorthand $C_{4}\left(r, t_{\mathrm{w}}\right)[16,35]$.

We characterize the spatial range of correlations through the coherence length:

$$
\xi_{k, k+1}\left(t_{\mathrm{w}}\right) \equiv I_{k+1}\left(t_{\mathrm{w}}\right) / I_{k}\left(t_{\mathrm{w}}\right),
$$

computed by means of the integrals

$$
I_{k}\left(t_{\mathrm{w}}\right) \equiv \int_{0}^{\infty} \mathrm{d} r r^{k} C_{4}\left(r, t_{\mathrm{w}}\right)
$$

Following recent work $[14,16,19,20,22]$, we shall focus our attention in the $k=1$ length estimate $\xi_{12}\left(t_{\mathrm{w}}\right)$.

Eventually, we have been able to equilibrate the system, in the sense that the integrals $I_{k}\left(t_{\mathrm{w}}\right)$ no longer depend on $t_{\mathrm{w}}$ (within errors). Of course, an infinite system never fully equilibrates. However, in the paramagnetic phase (and spin glasses in $D=2$ have only a paramagnetic phase at $T>0$ ), we can rather think of equilibration up to distance $r$ : for any fixed distance $r$ the $C_{4}\left(r, t_{\mathrm{w}}\right)$ approaches its equilibrium limit $C_{4}^{\mathrm{eq}}(r)$ exponentially fast in $t_{\mathrm{w}}$, after an $r$-dependent time threshold is reached; see appendix A.2. Given that the equilibrium propagator decays exponentially with distance, we can regard the system as equilibrated for all practical purposes once the $C_{4}\left(r, t_{\mathrm{w}}\right)$ equilibrates up to a distance of say $r=6 \xi_{12}^{\mathrm{eq}}(T)$. It is therefore meaningful to study numerically

$$
\xi_{12}^{\mathrm{eq}}(T)=\lim _{t_{\mathrm{w}} \rightarrow \infty} \xi_{12}\left(t_{\mathrm{w}}, T\right) .
$$

In our simulations, $\xi_{12}^{\mathrm{eq}}(T)$ ranges from $\xi_{12}^{\mathrm{eq}}(T=1.1) \approx 4.3$ to $\xi_{12}^{\mathrm{eq}}(T=0.5) \approx 39.4$ : this is why we expect that $L=512$ is large enough to accommodate $L \rightarrow \infty$ conditions $[14,19,22]$.

In fact, if one takes first the limit $L \rightarrow \infty$ and only afterwards goes to low $T$, we expect a critical point at $T=0$ :

$$
\xi_{12}^{\mathrm{eq}}(T) \sim T^{-\nu}+\ldots, \quad 1 / \nu=-\theta
$$

where the dots stand for (rather complex [29]) subleading corrections to scaling. The stiffness exponent $\theta$ has been computed in a $T=0$ simulation for Gaussian-distributed couplings, $\theta=-0.2793(3)$ [36] (the identity $-\theta=1 / \nu$ was already confirmed in former Gaussian coupling simulations; see for example [29, 37]). We have checked in [22] that equation (7) holds as well, with the same $\theta$, for our $J= \pm 1$ couplings.

Some readers may be unfamiliar with our coherence-length estimators, so let us relate our $\xi_{k, k+1}$ to the second-moment correlation length which is commonly studied in the context of equilibrium-critical phenomena [28, 38]. Let $\hat{C}_{4}\left(\boldsymbol{p}, t_{\mathrm{w}}\right)$ be the Fourier transform of $C_{4}\left(\boldsymbol{r}, t_{\mathrm{w}}\right)$. In the thermodynamic limit $L \rightarrow \infty$, the momentum $\boldsymbol{p}$ is a continuous variable. In the presence of rotational invariance (a reasonable assumption 
even for a fairly small $\left.\xi_{12}\left(t_{\mathrm{w}}\right)[16]\right), \hat{C}_{4}$ depends on the squared momentum $p^{2}$. Hence, the second moment correlation length is

$$
\xi^{2 \text { nd moment }}=\sqrt{-\left.\frac{\partial \log \hat{C}_{4}}{\partial p^{2}}\right|_{p^{2}=0}} .
$$

Equation (8) can be conveniently adapted to a finite lattice, hence discrete $\boldsymbol{p}[28,38$, 39], which partly explains its popularity. In real space, and assuming again $L \rightarrow \infty$ and rotational invariance, equation (8) reads in dimension $D$

$$
\xi^{\text {nd moment }, D}=\sqrt{\frac{1}{2 D} \frac{I_{D+1}}{I_{D-1}}}=\sqrt{\frac{\xi_{D-1, D} \xi_{D, D+1}}{2 D}} .
$$

The rationale for preferring $\xi_{12}$ over the more familiar $\xi^{2 \text { nd moment,D }}$ is a practical one [16]: statistical errors grow rapidly with the index $k$ of the requested integrals $I_{k}$.

For later use, we note as well that the (equilibrium) spin-glass susceptibility is

$$
\chi=\sum_{x, y=-\infty}^{\infty} C_{4}^{\mathrm{eq}}(x, y) \approx 2 \pi I_{1}^{\mathrm{eq}},
$$

where we have assumed again rotational invariance, as well as $\xi_{12}^{\mathrm{eq}} \gg 1$, in order to approximate the double summation by the integral $I_{1}^{\text {eq }}$ (in the general space dimension, $\left.\chi \propto I_{D-1}\right)$.

\section{On the spatial structure of the correlations}

In this section, we shall consider the Edwards-Anderson correlation function $C_{4}\left(r, t_{\mathrm{w}} ; T\right)$ as a function of distance, temperature and time. After some preliminary considerations, we shall address two different questions related to $C_{4}\left(r, t_{\mathrm{w}} ; T\right)$ : (i) how does the equilibrium correlation $C_{4}^{\mathrm{eq}}(r ; T)$ relate to the theory of a free field? (section 4 ); (ii) is the outof-equilibrium correlation function $C_{4}\left(r, t_{\mathrm{w}} ; T\right)$ given by free-field theory? (section 5 ).

Before addressing the above questions, let us frame the discussion. An underlying assumption in our analysis is that our choice of $k=1$ for $\xi_{k, k+1}$, recall equation (4), is immaterial $[14,16]$. This assumption is plausible because scale invariance suggests that the Edwards-Anderson correlation function behaves for large $r$ as

$$
C_{4}\left(r, t_{\mathrm{w}} ; T\right) \approx \frac{1}{r^{\zeta}} g\left[\frac{r}{l\left(t_{\mathrm{w}}, T\right)}, \frac{l\left(t_{\mathrm{w}}, T\right)}{l_{\mathrm{eq}}(T)}\right], \quad l_{\mathrm{eq}}(T)=\lim _{t_{\mathrm{w}} \rightarrow \infty} l\left(t_{\mathrm{w}}, T\right) .
$$

Unfortunately, we cannot extract the length scale $l\left(t_{\mathrm{w}}, T\right)$ because we do not have any a priori information on the scaling function $g$ in equation (11). This is why we use the integral estimators $\xi_{k, k+1}\left(t_{\mathrm{w}}\right)$, equation (4), that according to equation (11), are proportional to $l\left(t_{\mathrm{w}}, T\right)$ : 


$$
\xi_{k, k+1}\left(t_{\mathrm{w}}\right)=l\left(t_{\mathrm{w}}, T\right) \frac{\int_{0}^{\infty} \mathrm{d} x x^{k+1-\zeta} g(x, \hat{l})}{\int_{0}^{\infty} \mathrm{d} x x^{k-\zeta} g(x, \hat{l})}, \quad \hat{l}=\frac{l\left(t_{\mathrm{w}}, T\right)}{l_{\mathrm{eq}}(T)} .
$$

Equation (11) can be checked in the limiting case of an equilibrated system, $t_{\mathrm{w}} \rightarrow \infty$. Indeed, because we are in a paramagnetic phase (recall equation (7)), the renormalization group predicts that the Edwards-Anderson correlations are (asymptotically) given by the free-field propagator [27, 28]

$$
C_{4}^{\mathrm{eq}}(r ; T) \sim K_{0}\left[r / \xi_{\exp }(T)\right] \text { for } r \gg \xi_{\exp }(T) .
$$

In the above expression, which defines the so-called exponential correlation length $\xi_{\exp }(T), K_{0}$ is the 0th-order modified Bessel function of the second kind [40]. We remark that equation (13) is specific for $D=2$ (see appendix for general space dimension). After making the identification

$$
l_{\mathrm{eq}}(T) \equiv \xi_{\exp }(T)
$$

we see that equation (13) becomes a particular case of equation (11).

In order to investigate further equation (11), figure 1 shows the ratio of characteristic lengths $\xi_{23} / \xi_{12}$. Using equation (12) we obtain the expected behavior of the dimensionless ratio in the scaling limit (i.e. $\xi_{\exp }(T) \rightarrow \infty$ at fixed $l\left(t_{\mathrm{w}}, T\right) / \xi_{\exp }(T)$ ):

$$
\frac{\xi_{23}\left(t_{\mathrm{w}}, T\right)}{\xi_{12}\left(t_{\mathrm{w}}, T\right)}=\frac{\left[\int_{0}^{\infty} \mathrm{d} x x^{3-\zeta} g(x, \hat{l})\right]\left[\int_{0}^{\infty} \mathrm{d} x x^{1-\zeta} g(x, \hat{l})\right]}{\left[\int_{0}^{\infty} \mathrm{d} x x^{2-\zeta} g(x, \hat{l})\right]^{2}}, \hat{l}=\frac{l\left(t_{\mathrm{w}}, T\right)}{\xi_{\exp }(T)}
$$

The above expression unveils the role of $l\left(t_{\mathrm{w}}, T\right) / \xi_{\exp }(T)$. In fact, should the shape of the $r$-dependence in $C_{4}\left(r, t_{\mathrm{w}} ; T\right)$ be independent of time (thus, independent of $\left.l\left(t_{\mathrm{w}}, T\right) / \xi_{\exp }(T)\right)$, then $\xi_{23} / \xi_{12}$ would also be time-independent. Instead, we see in figure 1 that $\xi_{23} / \xi_{12}$ varies significantly as $\xi_{12}\left(t_{\mathrm{w}}\right)$ grows.

Of course, we knew beforehand that the shape of $C_{4}\left(r, t_{\mathrm{w}} ; T\right)$ must change with time: equation (13) tells us that $C_{4}^{\mathrm{eq}}(r ; T)$ decays exponentially: $C_{4}^{\mathrm{eq}}(r ; T) \sim \mathrm{e}^{-r / \xi_{\exp }} / \sqrt{r / \xi_{\exp }}$. Instead, the general arguments in appendix A.2 imply a super-exponential decay for the out-of-equilibrium correlation function, $C_{4}\left(r, t_{\mathrm{w}} ; T\right) \sim \mathrm{e}^{-(r / \hat{\xi})^{\beta}}$, with $\beta>1$. What figure 1 tells us is that the change in the functional form of $C_{4}\left(r, t_{\mathrm{w}} ; T\right)$ happens gradually.

However, there $i s$ something surprising in the large- $t_{\mathrm{w}}$ limit in figure 1. Barring high-temperature corrections, the equilibrium $\xi_{23} / \xi_{12}$ turns out to be compatible with $16 / \pi^{2}$, which is its free-field value (A.17). This is the first indication suggesting that equation (13) might work for $r \leqslant \xi_{\exp }$ as well, way before its natural validity range.

Let us now find a workaround on the annoying dependence on $l\left(t_{\mathrm{w}}, T\right) / \xi_{\exp }(T)$ in equation (12) (this dependency is a nuisance because, although $\xi_{\exp }(T)$ can be obtained from our data, see section $4, l\left(t_{\mathrm{w}}\right)$ remains a mystery). Fortunately, equation (12) suggests that the (computable) dimensionless ratio $\xi_{12}\left(t_{\mathrm{w}}, T\right) / \xi_{12}^{\mathrm{eq}}(T)$ is a one-to-one function of $l\left(t_{\mathrm{w}}, T\right) / \xi_{\exp }(T)$. Hence, we can compare out-of-equilibrium data at different temperatures by plotting $\xi_{23} / \xi_{12}$ as a function of $\xi_{12}\left(t_{\mathrm{w}}, T\right) / \xi_{12}^{\mathrm{eq}}(T)$, see figure 1 . Barring corrections for small $\xi_{12}^{\mathrm{eq}}(T)$ it is clear that the data collapse to a master curve, which is exactly what we expect from equation (12). We note as well that the same curve can be computed analytically for the free field (full curve in figure 1). The free-field 
Out-of-equilibrium 2D Ising spin glass: almost, but not quite, a free-field theory

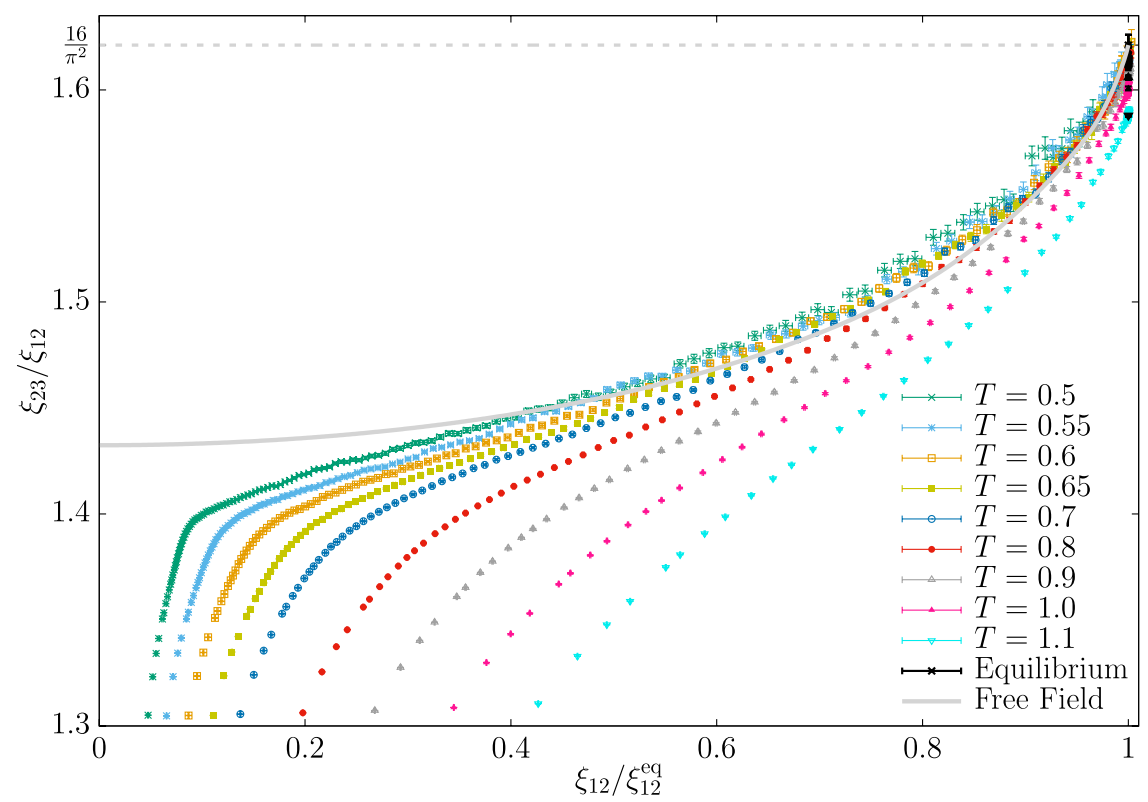

Figure 1. As time evolves (i.e. $\xi_{12}\left(t_{\mathrm{w}} ; T\right)$ grows until it reaches its equilibrium value $\xi_{12}^{\mathrm{eq}}(T)$ ), the scale-invariant ratio $\xi_{23}\left(t_{\mathrm{w}}, T\right) / \xi_{12}\left(t_{\mathrm{w}}, T\right)$ varies, which unveils the dependency on the unknown length scale $l\left(t_{\mathrm{w}}\right)$ in equations (11), (12) and (15). The figure shows that (barring small $\xi_{12}^{\mathrm{eq}}(T)$ corrections) the temperature dependence can be absorbed by plotting the data as a function of the scaleinvariant ratio $\xi_{12}\left(t_{\mathrm{w}}, T\right) / \xi_{12}^{\mathrm{eq}}(T)$. Indeed, in agreement with equation (15), our data collapse to a master curve when $\xi_{12}^{\mathrm{eq}}(T)$ grows upon lowering the temperature. An analogous master curve can be computed analytically for a non-interacting field (full line), see equations (A.15) and (A.17) in appendix A.1. Surprisingly, the master curve for the free field is a very good approximation for the Edwards-Anderson model. In fact, the free-field prediction might even be exact if the equilibrium limit $\xi_{12}\left(t_{\mathrm{w}}, T\right) / \xi_{12}^{\mathrm{eq}}(T) \rightarrow 1$ is taken first, and the scaling limit $\xi_{12}^{\mathrm{eq}}(T) \rightarrow \infty$ is taken afterwards.

master curve turns out to be fairly close to the limiting master curve for the EdwardsAnderson model.

We are now ready to address the questions posed at the beginning of this section.

\section{Equilibrium Edwards-Anderson correlations and the theory of a free field}

Let us consider the paramagnetic phase of a typical $D$-dimensional spin system in thermal equilibrium. The asymptotic behaviors of the correlation function are

$$
C^{\mathrm{eq}}\left(r \ll \xi_{\exp }\right) \sim \frac{1}{r^{D-2+\eta}}, \quad C^{\mathrm{eq}}\left(r \gg \xi_{\exp }\right) \sim \xi_{\exp }^{D-2-\eta} \frac{K_{Q}\left(r / \xi_{\exp }\right)}{\left(r / \xi_{\exp }\right)^{Q}},
$$

where $\eta$ is the anomalous dimension, $Q=(D-2) / 2$ and $K_{Q}$ is the $Q$ th-order modified Bessel function of the second kind [40]. The normalizations in equation (16) ensure that 
(i) $C^{\mathrm{eq}}(r=1) \sim 1$ (which is certainly the case for the Edwards-Anderson $C_{4}^{\mathrm{eq}}(r ; T)$ ), and (ii) the asymptotic behavior for small and large $r$ connects smoothly at $r=\xi_{\exp }{ }^{7}$.

However, let us take seriously for one minute the suggestion that the large-distance asymptotic behavior holds all the way down to $r \sim 1$. Now, specializing to $D=2$ and recalling that $K_{0}(y \rightarrow 0) \sim \log 1 / y$, we see that the condition $C^{\mathrm{eq}}(r=1) \sim 1$ implies that

$$
C_{2 \mathrm{D}, \text { non-standard }}^{\mathrm{eq}}(r) \sim \frac{K_{0}\left(r / \xi_{\exp }\right)}{\log \xi_{\exp }}
$$

Funnily enough, figure 1 suggests that the (equilibrium) 2D Ising spin glass could really follow the non-standard behavior in equation (17), even for $r<\xi_{\text {exp }}$. Our aim here will be to further explore this hypothesis.

Equation (17) suggests to start by fitting our equilibrium correlation function to

$$
C_{4}^{\mathrm{eq}}(r ; T)=\mathcal{A}\left(\xi_{\exp }\right)\left[K_{0}\left(\frac{r}{\xi_{\exp }(T)}\right)+K_{0}\left(\frac{L-r}{\xi_{\exp }(T)}\right)\right], \quad L=512,
$$

where $\mathcal{A}\left(\xi_{\text {exp }}\right)$ is an amplitude depending on temperature through $\xi_{\exp }(T)$. We have included in (18) the first image term, $K_{0}\left[(L-r) / \xi_{\exp }\right]$ (mind our periodic boundary conditions), as a further control of finite-size effects. In fact, the results turn out to vary by less than a tenth of an error bar (one standard deviation) when the image term is removed. This agreement confirms that the $L=\infty$ limit has been effectively reached.

The results of the fit to equation (18) are reported in table 1. As the reader may check, even in the most difficult case, namely $T=0.5, \xi_{\exp }(T)$ is computed with $1 \%$ accuracy. We find as well, see figure 2 top, that the consistency condition $C^{\mathrm{eq}}(r=1) \sim 1$ expressed in equation (17) is well satisfied by our data.

A further confirmation of equation (18) comes from the second-moment correlation length. Combining equation (9), as applied to $D=2$, with equation (A.14) we see that equation (18) implies

$$
\xi^{2 \text { nd moment,eq }}=\xi_{\text {exp }} .
$$

Thanks to previous results in [29], we may compare these two characteristic lengths, see tables 1 and 2 . The agreement is most satisfactory.

Of course, one cannot expect equation (18) to hold for all $r$. Indeed, the fit works only for $r \geqslant r_{\min }$, see table 1 . We find that the ratio $r_{\min } / \xi_{\exp }$ is small, but remains finite as $\xi_{\text {exp }}$ grows upon lowering $T$. In fact, we have empirically found that

$$
C_{4}^{\mathrm{eq}}(r ; T)-\mathcal{A}\left(\xi_{\exp }\right) K_{0}\left(r / \xi_{\exp }\right)=\mathcal{B}\left(\xi_{\exp }\right) \frac{\exp \left[-7 r / \xi_{\text {exp }}\right]}{\left(r / \xi_{\exp }\right)^{0.2}} .
$$

We have checked at $T=0.5$ and 0.55 that equation (20), for which we lack a theoretical justification, works for all $r \geqslant 1$ (in the sense of an acceptable $\chi^{2} /$ dof). Our standard regularity condition $C_{4}^{\mathrm{eq}}(r=1, T) \sim 1$ tells us that

\footnotetext{
${ }^{7}$ The $r \gg \xi_{\exp }$ asymptotic behavior in equation (16) has an additional factor $\xi^{-\eta}$ as compared with the free field, equation (A.4). This extra factor is the origin of the wave-function renormalization $Z_{\phi} \sim \xi^{\eta / 2}$ [27, 28], which for $\eta=0$ will produce a logarithmic divergence, see also the discussion of equation (17).
} 
Table 1. For each temperature in our simulations, we report the results of a fit to equation (18). Given that the numerical estimates of $C_{4}^{\mathrm{eq}}(r ; T)$ are dramatically correlated for different distances $r$, we use as the fit's figure of merit the diagonal $\chi^{2}$ (i.e. the $\chi^{2}$ statistics as computed, keeping only the diagonal terms in the covariance matrix). These correlations are responsible for the anomalously low $\chi^{2}$ that we find. The distances included in the fit are $r_{\min } \leqslant r \leqslant r_{\max }$ (see [22] for details). To compute errors in the fit parameters, namely $\mathcal{A}\left(\xi_{\exp }\right)$ and $\xi_{\exp }$, we employ the jackknife as implemented in [41]: we fit for each jackknife block (using for all blocks the diagonal covariance matrix), and compute errors from the blocks' fluctuations. We also report the ratio $\xi_{12}^{\mathrm{eq}} / \xi_{\exp }$ (in order to account for statistical correlations, errors were computed with the jackknife). In a freefield theory, $\xi_{12}^{\mathrm{FF}, \mathrm{eq}} / \xi_{\exp }=\pi / 2=1.5707963 \ldots$, see equation (A.14), which is fairly close to our numerical results for the Edwards-Anderson model. The behavior of $\xi_{12}^{\mathrm{eq}} / \xi_{\exp }$ in the limit of large $\xi_{\exp }$ is studied in figure 2 bottom.

\begin{tabular}{lccllll}
\hline$T$ & $r_{\min }$ & $r_{\max }$ & $\chi^{2} /$ dof & $A\left(\xi_{\exp }\right)$ & \multicolumn{1}{l}{$\xi_{\exp }$} & $\xi_{12}^{\text {eq }} / \xi_{\exp }$ \\
\hline 0.50 & 19 & 202 & $13.72 / 182$ & $0.2295(34)$ & $24.98(30)$ & $1.5758(27)$ \\
0.55 & 14 & 166 & $70.53 / 151$ & $0.2469(27)$ & $18.10(16)$ & $1.5757(24)$ \\
0.60 & 10 & 142 & $36.85 / 131$ & $0.2655(20)$ & $13.63(8)$ & $1.5771(21)$ \\
0.65 & 8 & 113 & $47.37 / 104$ & $0.2812(19)$ & $10.68(6)$ & $1.5772(16)$ \\
0.70 & 8 & 103 & $62.60 / 94$ & $0.2981(20)$ & $8.59(4)$ & $1.5815(22)$ \\
0.80 & 5 & 66 & $22.82 / 60$ & $0.3259(12)$ & $5.942(17)$ & $1.5798(9)$ \\
0.90 & 3 & 50 & $35.97 / 46$ & $0.3566(7)$ & $4.358(8)$ & $1.5841(5)$ \\
1.00 & 4 & 39 & $5.38 / 34$ & $0.3867(10)$ & $3.355(6)$ & $1.5893(8)$ \\
1.10 & 4 & 31 & $9.70 / 26$ & $0.4189(11)$ & $2.671(4)$ & $1.5994(9)$ \\
\hline
\end{tabular}

$$
\mathcal{B}\left(\xi_{\exp }\right) \sim\left[\frac{1}{\xi_{\exp }}\right]^{0.2} \text { for } \quad \xi_{\exp } \rightarrow \infty .
$$

We are finally ready to consider the extrapolation to large $\xi_{\exp }$ of the ratios $\xi_{k, k+1}^{\mathrm{eq}} / \xi_{\exp }$. We shall start by dividing the $\xi_{k, k+1}^{\mathrm{eq}} / \xi_{\exp }$ by their free-field value in equation (A.14):

$$
J_{k} \equiv \frac{\xi_{k, k+1}^{\mathrm{eq}}}{\xi_{\exp }} \frac{1}{2} \frac{\Gamma^{2}[(k+1) / 2]}{\Gamma^{2}[(k+2) / 2]} .
$$

Our working hypothesis is that $J_{k} \rightarrow 1$ for large $\xi_{\text {exp }}$. Then, a straightforward computation starting from equations (20) and (21) predicts that the finite- $\xi_{\exp }$ corrections for $\xi_{k, k+1}^{\mathrm{eq}}=I_{k+1}^{\mathrm{eq}} / I_{k}^{\mathrm{eq}}$ take the form of a series expansion in the corrections-to-scaling function

$$
v\left(\xi_{\exp }\right)=\frac{1}{\left(\xi_{\exp }\right)^{0.2} \mathcal{A}\left(\xi_{\exp }\right)} .
$$

In addition, we have the standard corrections in $1 / \xi_{\exp }$, stemming from our consideration of continuous functions of $r / \xi_{\exp }$ while numerical data can be obtained only for integer $r$. Accordingly, we have fitted our data to

$$
J_{k}=1+a_{k} v\left(\xi_{\exp }\right)+\frac{b_{k}^{(1)}}{\xi_{\exp }}+\frac{b_{k}^{(2)}}{\xi_{\exp }^{2}},
$$


Out-of-equilibrium 2D Ising spin glass: almost, but not quite, a free-field theory

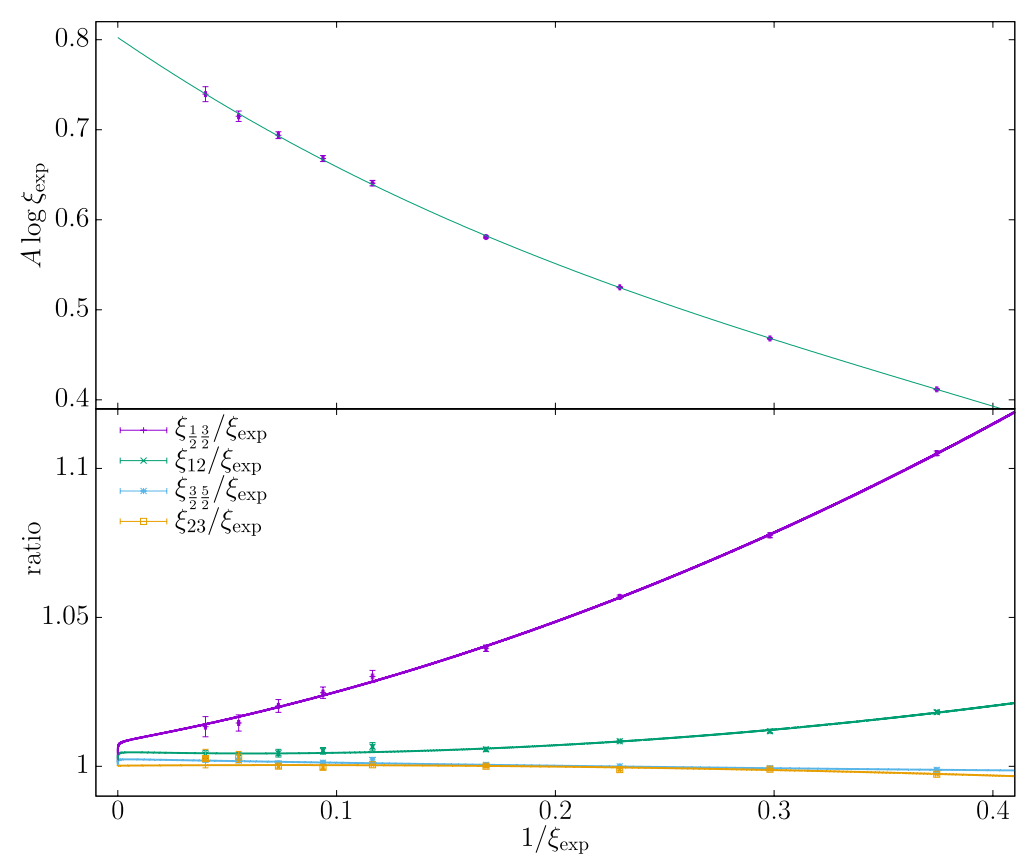

Figure 2. Top: the consistency condition $C^{\mathrm{eq}}(r=1) \sim 1$, see equation (17), requires the amplitude in the fit in equation (18) to scale as $\mathcal{A}\left(\xi_{\exp } \rightarrow \infty\right) \sim 1 / \log \xi_{\exp }$. Indeed, the plot shows that $\mathcal{A}\left(\xi_{\exp }\right) \log \xi_{\exp }$ is excellently represented, $\chi^{2} /$ dof $=2.2 / 5$, by a cubic polynomial in $1 / \xi_{\text {exp }}$, implying $\mathcal{A}\left(\xi_{\exp }\right) \log \xi_{\exp } \approx 0.8$ for large $\xi_{\text {exp }}$. Bottom: if the non-standard scaling (17) holds true, all the $J_{k}$ defined in equation (22) should tend to 1 when $\xi_{\exp } \rightarrow \infty$ (the $J_{k}$ are the Edwards-Anderson $\xi_{k, k+1}^{\mathrm{eq}} / \xi_{\exp }$ divided by their free-field counterparts). We show $J_{k}$ as a function of $1 / \xi_{\exp }$, for $k=1 / 2,1,3 / 2$ and 2. Lines are fits to equation (24) (note that the function $v\left(\xi_{\exp }\right)$, equation (23), is continuous, but has an infinite slope at $\frac{1}{\xi_{\exp }}=0$ ). The corresponding figures of merit of these are $\chi^{2} /$ dof $=2.4 / 6(k=1 / 2), \chi^{2} /$ dof $=4.0 / 6(k=1), \chi^{2} /$ dof $=3.9 / 6$ $(k=3 / 2)$ and $\chi^{2} /$ dof $=10.0 / 6(k=2)$.

Table 2. Second moment correlation length in equilibrium as computed in an $L=128$ system by means of a parallel tempering simulation (data from [29]). We expect $\xi^{2 \text { nd moment,eq }}=\xi_{\text {exp }}$, see the discussion of equation (19). In fact, leaving aside $T=0.5$ (because an $L=128$ lattice is clearly too small to represent the $L \rightarrow \infty$ limit for that temperature), the agreement with the corresponding values for $\xi_{\exp }$ in table 1 is impressive.

\begin{tabular}{lc}
\hline$T$ & $\xi^{2 \text { nd moment,eq }}$ \\
\hline 0.50 & $23.99(17)$ \\
0.55 & $17.95(11)$ \\
0.65 & $10.753(39)$ \\
0.60 & $13.712(65)$ \\
0.70 & $8.649(26)$ \\
0.80 & $5.968(13)$ \\
0.90 & $4.3854(71)$ \\
1.00 & $3.3657(45)$ \\
1.10 & $2.6782(51)$ \\
\hline
\end{tabular}


with fitting parameters $a_{k}, b_{k}^{(1)}$ and $b_{k}^{(2)}$. We have found fair fits to equation (24), see figure 2 bottom, even for $k$ as small as $k=1 / 2$ (the smaller the $k$, the more highlighted the small- $r$ region). $\mathcal{A}\left(\xi_{\exp }\right)$ is promoted to a continuous function of $\xi_{\exp }$ through the fit in figure 2 top (this is needed to compute $v\left(\xi_{\exp }\right)$ ). To assess the relative importance of the correction terms in equation (24), we may consider $b_{k}^{(1)} / a_{k}$ : in figure 2 bottom, these ratios of amplitudes are $b_{1 / 2}^{(1)} / a_{1 / 2} \approx 40, b_{1}^{(1)} / a_{1} \approx 6.6, b_{3 / 2}^{(1)} / a_{3 / 2} \approx 11$, and $b_{2}^{(1)} / a_{2} \approx 52$.

Notice that the equilibrium second-moment correlation length was computed in [30] (which coincides with $\xi_{\exp }$, see equation (19) and table 2), as well as the spin-glass susceptibility, recall equation (10). A very large value of $\xi_{\exp } \approx 200$ was reached thanks to a combination of parallel tempering, cluster methods and finite-size scaling [30]. However, the scaling of $\chi$ was barely under control, in spite of the very large $\xi_{\text {exp }}$. The short-distances behavior identified in equations (20) and (21) explains this difficulty. Indeed, using the equivalence $\chi=2 \pi I_{1}^{\text {eq }}$, only valid in $D=2$, one easily finds that

$$
\chi=\xi^{2}\left[b_{\chi}^{0}+a_{\chi} v\left(\xi_{\exp }\right)+\frac{b_{\chi}^{(1)}}{\xi_{\exp }}+\frac{b_{\chi}^{(2)}}{\xi_{\exp }^{2}}+\frac{b_{\chi}^{(3)}}{\xi_{\exp }^{3}}+\ldots\right],
$$

where $a_{\chi}$ and the $b_{\chi}^{(i)}$ are scaling amplitudes. A fair fit to equation (25) is shown in the full line in figure 3. The width of that full line has been chosen to correspond with the error bars, while the dotted line in figure 3 is the leading term $\chi \sim b_{\chi}^{(0)} \xi_{\text {exp }}^{2}$. We see in figure 3 that the full and the dotted lines coalesce only for $\xi_{\exp }>100$, in nice agreement with the results found in [30].

In summary, in the scaling limit $\xi_{\text {eq }} \rightarrow \infty$, the equilibrium correlation function for the Ising spin glass seems to follow the non-standard scaling in equation (17). However, some readers may consider far-fetched our parameterization of short-distances corrections to the free-field propagator in equations (20) and (21). These skeptical readers may keep the more conservative conclusion that violations to the free-field prediction $J_{k}\left(\xi_{\text {eq }} \rightarrow \infty\right)=1$ are, at most, of $0.3 \%$ for $k=1 / 2,1,3 / 2$, and 2 .

\section{Out-of-equilibrium Edwards-Anderson correlations and the theory of a free field}

Relating the Langevin dynamics of a free field with spin-glass dynamics may seem surprising at first sight. Indeed, the dynamics of a spin glass in its paramagnetic phase may be characterized through a scaling function [22]

$$
\frac{\xi_{12}\left(t_{\mathrm{w}}, T\right)}{\xi_{12}^{\mathrm{eq}}(T)}=\mathcal{F}\left(\frac{t_{\mathrm{w}}}{\tau(T)}\right)+\mathcal{O}\left(\left[\xi_{12}\left(t_{\mathrm{w}}, T\right)\right]^{-\omega},\left[\xi_{12}^{\mathrm{eq}}(T)\right]^{-\omega}\right)
$$

where exponent $\omega$ controls corrections to scaling, $\tau(T)$ is a characteristic time scale, and the dynamics at short times are described by a dynamic exponent $\hat{z}$ :

$$
\mathcal{F}(x \rightarrow 0) \propto x^{1 / \hat{z}} .
$$


Out-of-equilibrium 2D Ising spin glass: almost, but not quite, a free-field theory

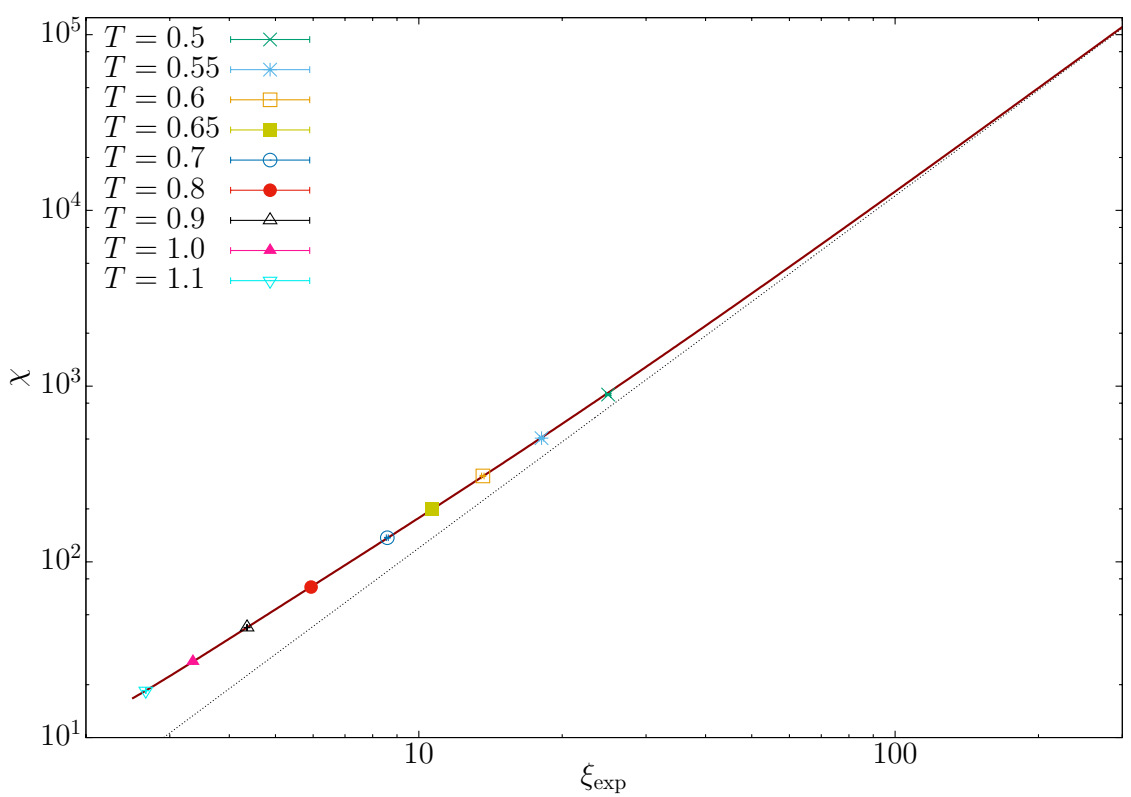

Figure 3. Equilibrium spin glass susceptibility, $\chi=2 \pi I_{1}^{\mathrm{eq}}$, see equation (10), as a function of $\xi_{\exp }$. The full line is a fit to equation (25) $\left(\chi^{2} / \mathrm{dof}=2.8 / 5\right)$. One could set $b_{\chi}^{(3)}=0$ in equation (25), but at the price of including only data with $\xi_{\exp }>4$ in the fit (in such a case, one finds $\chi^{2} /$ dof $=3.6 / 4$ ). The dotted line is the dominant term in equation (25), $\chi \sim b_{\chi}^{(0)} \xi_{\exp }^{2}$. The horizontal and vertical ranges of the plot have been chosen to match those of figure 2 in [30].

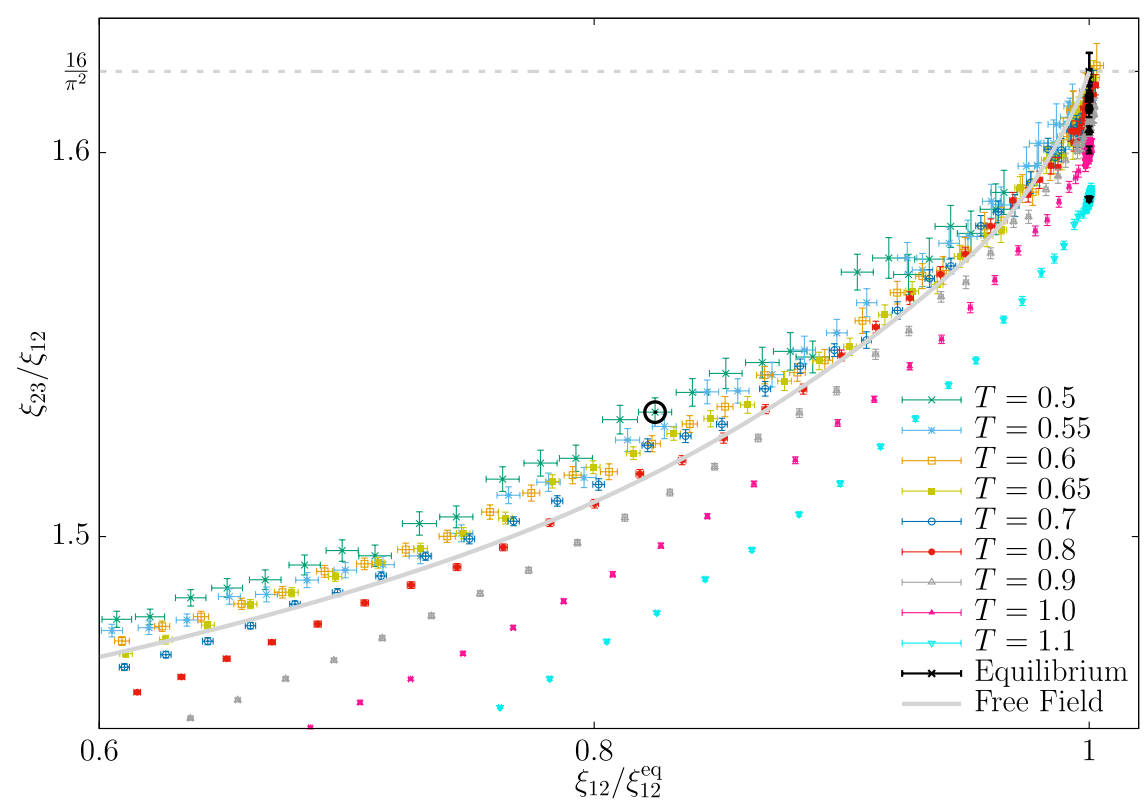

Figure 4. Zoom of data in figure 1 . The scaling limit $\xi_{\exp }(T) \rightarrow \infty$ at fixed $\xi_{12}\left(t_{\mathrm{w}}, T\right) / \xi_{12}^{\mathrm{eq}}(T)$ slightly differs for the Edwards-Anderson model (data points) and for the non-interacting field (full line). However, disentangling the two models' behavior becomes difficult upon approaching equilibrium, $\xi_{12}\left(t_{\mathrm{w}}, T\right) / \xi_{12}^{\mathrm{eq}}(T) \rightarrow 1$. The time $t_{\mathrm{w}}$ which is explicitly compared with the free-field model in figure 5 is marked by a circle (for $T=0.5$ ). 
Out-of-equilibrium 2D Ising spin glass: almost, but not quite, a free-field theory

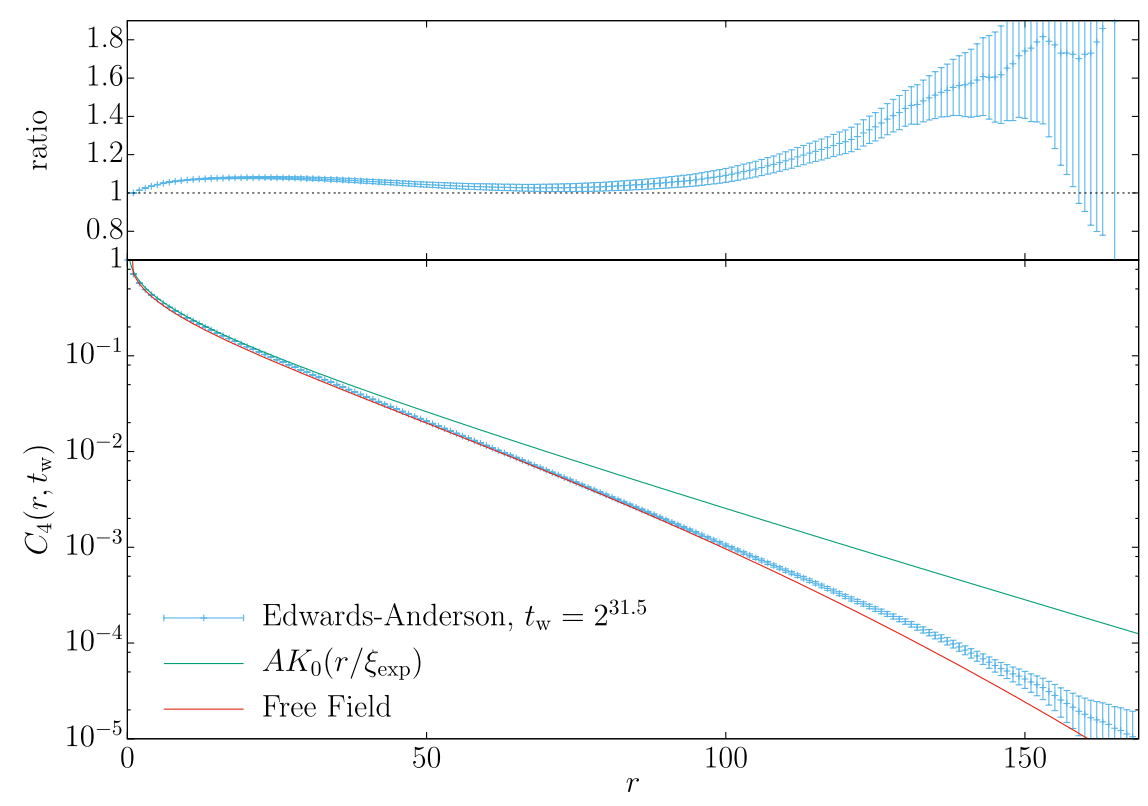

Figure 5. Main: for $T=0.5$, we compare the Edwards-Anderson correlation function $C_{4}\left(r, t_{\mathrm{w}}\right)$ with its free-field counterpart $G\left(r, t_{\mathrm{w}}^{\mathrm{FF}}\right)$, see equation (A.3). To match the parameters for the free field, we fix $\xi_{12}^{\mathrm{FF}}\left(t_{\mathrm{w}}^{\mathrm{FF}}\right) / \xi_{12}^{\mathrm{FF}, \text { eq }}=0.824585$, the value pinpointed by the circle in figure 4 , and take $\xi_{\exp }(T=0.5)$ from table 1 . The overall normalization of the free field is chosen to have $C_{4}\left(r=1, t_{\mathrm{w}}\right)=G\left(r=1, t_{\mathrm{w}}^{\mathrm{FF}}\right)$. We also compare the two propagators with the asymptotic form of the equilibrium EdwardsAnderson correlation function, $\mathcal{A}\left(\xi_{\exp }\right) K_{0}\left(r / \xi_{\exp }\right)$. Top: ratio $C_{4}\left(r, t_{\mathrm{w}}\right) / G\left(r, t_{\mathrm{w}}^{\mathrm{FF}}\right)$ as a function of $r$.

We have found empirically $\hat{z} \approx 7$ for the Edwards-Anderson model [22].

The analogous exponent for the free field is $\hat{z}_{\mathrm{FF}}=2$ (appendix A.1). The obvious, hardly surprising conclusion is that spin-glass dynamics are enormously slower than free-field dynamics. However, one may synchronize clocks between these two wildly differing systems by requiring (superscripts FF stand for free field)

$$
f=\frac{\xi_{12}\left(t_{\mathrm{w}}, T\right)}{\xi_{12}^{\mathrm{eq}}(T)}=\frac{\xi_{12}^{\mathrm{FF}}\left(t_{\mathrm{w}}^{\mathrm{FF}}\right)}{\xi_{12}^{\mathrm{FF}, \mathrm{eq}}} .
$$

This clock synchronization was implicitly performed in figure 1. We zoom this figure in figure 4 making it clear that the clock synchronization works only approximately: the free field and the Edwards-Anderson limit behave in the same way only in the limit of a system in thermal equilibrium.

In order to further expose the difference, in figure 5 we compare the EdwardsAnderson model correlation function $C_{4}\left(r, t_{\mathrm{w}} ; T\right)$ with its free-field counterpart in figure 5, after the appropriate parameter matching. It is clear that, even setting the same $\xi_{\exp }$ for both models and synchronizing the clocks as in equation (28), the freefield propagator has a higher curvature, as a function of $r$. 


\section{Conclusions}

We have studied the out-of-equilibrium dynamics of the 2D Edwards-Anderson model with binary couplings. We have been able to study the full range of dynamics from the initial transients to the equilibrium through numerical simulations with a time span of 11 orders of magnitude. We have considered the spatial dependence of the EdwardsAnderson correlation function $C_{4}\left(r, t_{\mathrm{w}}\right)$, that has been compared with the propagator of a free-field theory. Much to our surprise, we found that, after an appropriate clock synchronization between the two models, the free-field propagator provides a very good approximation to $C_{4}\left(r, t_{\mathrm{w}}\right)$ in the out-of-equilibrium regime. Furthermore, in the scaling limit $\xi_{12}^{\mathrm{eq}}$ for the equilibrium regime, after a logarithmic wavefunction renormalization, we find it extremely difficult to distinguish the two models numerically.

\section{Acknowledgments}

We thank Professor Gabriel Álvarez for discussions. This project has received funding from the European Research Council (ERC) under the European Unions Horizon 2020 research and innovation program (grant agreement No. 694925). We were partially supported by MINECO (Spain) through Grant Nos. FIS2015-65078-C2 and FIS201676359-P, and by the Junta de Extremadura (Spain) through Grant Nos. GRU10158, GR18079 and IB16013 (these five contracts were partially funded by FEDER). Our simulations were carried out at the BIFI supercomputing center (using the Memento and Cierzo clusters) and at the ICCAEx supercomputer center in Badajoz (Grinfishpc and Iccaexhpc). We thank the staff at the BIFI and ICCAEx supercomputing centers for their assistance.

\section{Appendix. The out-of-equilibrium dynamics of the free scalar field}

The Edwards-Anderson model in spatial dimension $D=2$ lies within its paramagnetic phase at all positive temperatures. Therefore, the relevant renormalization group fixed point is the one of the free scalar field (see e.g. [27, 28]). This observation implies that, at least in equilibrium, the free-field fixed point rules the system behavior at distances $r \gg \xi_{\text {eq. }}$.

However, the $D=2$ Edwards-Anderson model and the free-field theory might differ for distances $r \sim \xi_{\text {eq }}$. Furthermore, at these length scales, the two theories should be compared both under equilibrium and out-of-equilibrium conditions. In order to confront the two models, we compute here for the free field the same quantities that were studied for the Edwards-Anderson model in the main text.

Our starting point are the Langevin dynamics for a free field [27]. At the initial time, the field is fully disordered. The two-body correlation function $G\left(\boldsymbol{r}, t_{\mathrm{w}}\right)$ is analogous in the free-field theory of the Edwards-Anderson correlation function $C_{4}\left(\boldsymbol{r}, t_{\mathrm{w}}\right)$, recall equation (3). We can compute explictly the free field $G$ in Fourier space

$$
\hat{G}\left(\boldsymbol{p}, t_{\mathrm{w}}\right)=\frac{\left(1-\exp \left[-2 t_{\mathrm{w}}\left(p^{2}+\xi_{\exp }^{-2}\right)\right]\right)}{p^{2}+\xi_{\exp }^{-2}} .
$$


The above expression defines the so-called exponential correlation length, $\xi_{\exp }$ (indeed, $\hat{G}\left(\boldsymbol{p}, t_{\mathrm{w}}\right)$ tends to the Gaussian propagator $1 /\left(p^{2}+\xi_{\exp }^{-2}\right)$ in the limit of large $\left.t_{\mathrm{w}}\right)$. Note as well that there are two characteristic lengths in equation (A.1), namely the correlation length $\xi_{\text {exp }}$ and the diffusion length $\sqrt{t_{\mathrm{w}}}$. Thus, before starting our computation, it will be useful to introduce dimensionless length $(\boldsymbol{y})$ and time variables $(w)$ :

$$
\boldsymbol{y}=\boldsymbol{r} / \xi_{\exp }, \quad w=2 t_{\mathrm{w}} / \xi_{\exp }^{2} .
$$

Rotational invariance implies that the propagator will depend only on the length $y$ of vector $\boldsymbol{y}$ (on a lattice, rotational invariance is recovered only in the continuum limit $\xi_{\exp } \rightarrow \infty$ [27]; in the context of out-of-equilibrium spin glasses, the recovery of rotational invariance was investigated in [16]).

A straightforward computation (appendix A.3) allows us to transform back equation (A.1) from Fourier to real space:

$$
G\left(\boldsymbol{r}, t_{\mathrm{w}}\right)=\xi_{\exp }^{2-D} F_{D}(y, w), F_{D}(y, w)=\frac{1}{(4 \pi)^{D / 2}} \int_{0}^{w} \mathrm{~d} s \frac{\exp \left[-s-\frac{y^{2}}{4 s}\right]}{s^{D / 2}} .
$$

Armed with equation (A.3) we can compute, using the free field analogous theory, the $I_{k}\left(t_{\mathrm{w}}\right)$ integrals defined in equation (5). This computation is performed in appendix A.1. Equation (A.3) also simplifies the discussion of the large $y$ limit taken at fixed $w$ (appendix A.2).

The opposite limit, $w \rightarrow \infty$ for fixed $y$, yields the (equilibrium) Gaussian propagator (see [27] for further details):

$$
G^{\mathrm{eq}}(\boldsymbol{r})=\frac{\xi_{\exp }^{2-D}}{(4 \pi)^{D / 2}} \int_{0}^{\infty} \mathrm{d} s \frac{\exp \left[-s-\frac{y^{2}}{4 s}\right]}{s^{D / 2}}=\frac{\xi_{\exp }^{2-D}}{(2 \pi)^{D / 2}} \frac{K_{Q}(y)}{y^{Q}},
$$

where $Q=(D-2) / 2$ and $K_{Q}$ is the $Q$ th-order modified Bessel function of the second kind [40]. The large and small- $y$ behavior for $D>2$ is

$$
G^{\mathrm{eq}}\left(\boldsymbol{r} / \xi_{\exp } \rightarrow \infty\right) \sim \frac{\mathrm{e}^{-y}}{y^{(D-1) / 2}}, \quad G^{\mathrm{eq}}\left(\boldsymbol{r} / \xi_{\exp } \rightarrow 0\right) \sim \frac{1}{y^{D-2}}
$$

The neighborhood of $y \rightarrow 0$ for the case $D=2$ deserves special care:

$$
G^{\mathrm{eq}}\left(\boldsymbol{r} / \xi_{\exp } \rightarrow 0\right) \sim \log \frac{1}{y}
$$

\section{A.1. Integral estimators of dynamic correlations}

In analogy with equation (5), we shall characterize the free-field propagator through its moments (the superindex FF stands for free field)

$$
I_{k}^{\mathrm{FF}}\left(t_{\mathrm{w}}\right)=\int_{0}^{\infty} \mathrm{d} r r^{k} G\left(r, t_{\mathrm{w}}\right)
$$

where we have exploited the isotropy of the free-field propagator. We shall specialize to $D=2$, and compute the moments for a propagator of the form 


$$
G\left(r, t_{\mathrm{w}}\right)=\mathcal{A} \int_{0}^{w} \mathrm{~d} s \frac{\exp \left[-s-\frac{y^{2}}{4 s}\right]}{s},
$$

recall equations (A.2) and (A.3). In particular, equation (A.3) implies for the amplitude $\mathcal{A}=1 /(4 \pi)$. However, the main results in this section will be $\mathcal{A}$-independent (in particular, $\mathcal{A}$ could depend on $\xi_{\text {exp }}$ or $\left.w\right)$. We find

$$
\begin{aligned}
I_{k}^{\mathrm{FF}}\left(t_{\mathrm{w}}\right) & =\mathcal{A} \xi_{\exp }^{k+1} \int_{0}^{\infty} \mathrm{d} y y^{k} \int_{0}^{w} \mathrm{~d} s \frac{\exp \left[-s-\frac{y^{2}}{4 s}\right]}{s}, \\
& =\mathcal{A} \xi_{\exp }^{k+1} 2^{k} \Gamma[(k+1) / 2] \gamma[(k+1) / 2, w],
\end{aligned}
$$

by interchanging the ordering of the $y$ and $s$ integrals. In the above expression, $\Gamma(x)$ is Euler's Gamma function and $\gamma(x, w)$ is the lower incomplete Gamma function

$$
\gamma(x, w)=\int_{0}^{w} \mathrm{~d} s s^{x-1} \mathrm{e}^{-s}, \quad \gamma(x, w \rightarrow \infty)=\Gamma(x)+\mathcal{O}\left(w^{x-1} \mathrm{e}^{-w}\right) .
$$

For later use, we recall its small- $w$ behavior:

$$
\gamma(x, w \rightarrow 0)=\frac{w^{x}}{x}+\mathcal{O}\left(w^{x+1}\right) .
$$

The $\xi_{k, k+1}^{\mathrm{FF}}\left(t_{\mathrm{w}}\right)$ estimate of the size of the coherence length, recall equation (4), is

$$
\xi_{k, k+1}^{\mathrm{FF}}\left(t_{\mathrm{w}}\right) \equiv \frac{I_{k+1}^{\mathrm{FF}}\left(t_{\mathrm{w}}\right)}{I_{k}^{\mathrm{FF}}\left(t_{\mathrm{w}}\right)}=2 \frac{\Gamma[(k+2) / 2]}{\Gamma[(k+1) / 2]} \frac{\gamma[(k+2) / 2, w]}{\gamma[(k+1) / 2, w]} \xi_{\text {exp }} .
$$

The equilibrium limit, $w \rightarrow \infty$, is approached exponentially in $w$ (equation (A.11)):

$$
\xi_{k, k+1}^{\mathrm{FF}, \mathrm{eq}}=2 \frac{\Gamma^{2}[(k+2) / 2]}{\Gamma^{2}[(k+1) / 2]} \xi_{\text {exp }} .
$$

In other words, the integral estimators of the coherence length, in equilibrium but also out of equilibrium (at fixed $w$ ), are proportional to the exponential correlation length $\xi_{\text {exp }}$.

In the main text, we paid major attention to the approach to equilibrium of $\xi_{12}$ as computed in the Edwards-Anderson model. The free field analogous of equation (26) is

$$
\frac{\xi_{12}^{\mathrm{FF}}\left(t_{\mathrm{w}}\right)}{\xi_{12}^{\mathrm{FF}, \mathrm{eq}}}=\frac{\gamma(3 / 2, w)}{\Gamma(3 / 2)} \frac{\Gamma(1)}{\gamma(1, w)} .
$$

It is remarkable that equation (A.15) conforms exactly to the ansatz expressed for the Edwards-Anderson model in equation (26). Furthermore, because $w=2 t_{\mathrm{w}} / \xi_{\exp }^{2}(T)$, we find $\tau_{\mathrm{FF}}(T)=\xi_{\exp }^{2}(T) / 2$ for the free field analogous of the time scale in equation (26).

We can also compute the free-field exponent $\hat{z}_{\mathrm{FF}}$, recall equation (27), from the small- $w$ expansion of equation (A.15) (recall equation (A.12)):

$$
\frac{\xi_{12}^{\mathrm{FF}}\left(t_{\mathrm{w}}\right)}{\xi_{12}^{\mathrm{FF}, \mathrm{eq}}}=\frac{2 \sqrt{\pi}}{3} w^{1 / 2}+\mathcal{O}\left(w^{3 / 2}\right)
$$


which implies for the free field $\hat{z}_{\mathrm{FF}}=2$. The reader may check from equations (A.12)(A.14) that the small- $w$ behavior is $\xi_{k, k+1}^{\mathrm{FF}}\left(t_{\mathrm{w}}\right) / \xi_{k, k+1}^{\mathrm{FF}, \mathrm{eq}} \sim \sqrt{w}$ for any $k$, hence the result $\hat{z}_{\mathrm{FF}}=2$ is $k$-independent. Because $\hat{z}_{\mathrm{FF}}=2$ is rather smaller than the $\hat{z} \approx 7$ value that we found numerically for the Edwards-Anderson model, we conclude that the dynamics for the Edwards-Anderson model are enormously slower than the free-field Langevin dynamics, which is hardly surprising.

Nevertheless, equation (A.15) shows that $\xi_{12}^{\mathrm{FF}}\left(t_{\mathrm{w}}\right) / \xi_{12}^{\mathrm{FF}, \mathrm{eq}}$ is a monotonously increasing function of $w$. Hence, one can parameterize the free-field dynamics in terms of $\xi_{12}^{\mathrm{FF}}\left(t_{\mathrm{w}}\right) / \xi_{12}^{\mathrm{FF}, \mathrm{eq}}$, rather than $w$. In this way, we can obtain a meaningful comparison of the free field with the Edwards-Anderson dynamics. The quantities compared are dimensionless ratios such as $\xi_{k, k+1}\left(t_{\mathrm{w}}\right) / \xi_{\text {exp }}$ (its value for the free field is given in equation (A.13)), or in terms of ratios not involving $\xi_{\exp }$ such as $\xi_{23}\left(t_{\mathrm{w}}\right) / \xi_{12}\left(t_{\mathrm{w}}\right)$, recall figure 1. From equation (A.13), we easily find

$$
\frac{\xi_{23}^{\mathrm{FF}}\left(t_{\mathrm{w}}\right)}{\xi_{12}^{\mathrm{FF}}\left(t_{\mathrm{w}}\right)}=\frac{4}{\pi} \frac{\gamma(2, w) \gamma(1, w)}{\gamma^{2}(3 / 2, w)} .
$$

The limiting values are $\xi_{23}^{\mathrm{FF}} / \xi_{12}^{\mathrm{FF}}=9 /(2 \pi)($ for $w \rightarrow 0)$, and $\xi_{23}^{\mathrm{FF}} / \xi_{12}^{\mathrm{FF}}=16 / \pi^{2}($ for $w \rightarrow \infty)$.

\section{A.2. Asymptotic behavior of $F_{D}(y, w)$ (large $y$ at fixed $\left.w\right)$}

For any finite fixed-time $t_{\mathrm{w}}$, the free-field propagator in Fourier space, $\hat{G}\left(\boldsymbol{p}, t_{\mathrm{w}}\right)$ see equation (A.1), is an analytic function in the whole complex plane of the variable $p^{2}$. It follows that the function $F_{D}(y, w)$, defined in equation (A.3), tends to zero at large $y$ faster than $\mathrm{e}^{-A y}$ for any $A>0$ (a simply exponential decay corresponds with a pole singularity at $\left.p^{2}=-A^{2}[27]\right)$. This statement is in apparent contradiction with the asymptotic behavior in equation (A.5) which is exact, but only for $t_{\mathrm{w}}=\infty$. The way out of the paradox is simple: $\hat{G}\left(p^{2}=-\xi_{\exp }^{2}, t_{\mathrm{w}}\right)=2 t_{\mathrm{w}}$ which becomes a pole singularity only in the $t_{\mathrm{w}} \rightarrow \infty$ limit. It is clear that, at finite $t_{\mathrm{w}}$, some sort of crossover phenomenon is present. In this section we aim to discuss this crossover.

We start from the integral representation (A.3)

$$
\begin{aligned}
& F_{D}(y, w)=\frac{1}{(4 \pi)^{D / 2}} \int_{0}^{w} \mathrm{~d} s \mathrm{e}^{\Psi_{D}(s, y)}, \\
& \Psi_{D}(s, y)=-\frac{y^{2}}{4 s}-s-\frac{D}{2} \log s .
\end{aligned}
$$

Consider the function $\Psi_{D}(s, y)$ at fixed $y . \Psi_{D}(s, y)$ tends to $-\infty$ both for $s \rightarrow 0, \infty$. These two asymptotic behaviors of $\Psi_{D}(s, y)$ are separated by a maximum at

$$
s^{*}(y)=\frac{y^{2}}{D+\sqrt{D^{2}+4 y^{2}}} .
$$

Note that $s^{*}(y \rightarrow 0) \sim y^{2}$, but $s^{*}(y \rightarrow \infty) \sim y / 2$.

Now, imagine that we hold $y$ fixed ( $y$ should be large enough to have $s^{*}(y) \approx y / 2$ to a good approximation). If $w \gg s^{*}(y)$ we can estimate $F_{D}(y, w)$ through a straightforward 
saddle-point expansion around $s^{*}(y)$ that reproduces the $w=\infty$ asymptotic behavior in equation (A.5):

$$
F_{D}^{(\mathrm{SP})}(y, w) \sim \frac{\mathrm{e}^{-y}}{y^{(D-1) / 2}}
$$

The error induced by the finite $w$ is $\sim \mathrm{e}^{\Psi_{D}(w, y)} /\left|\partial_{\mathrm{w}} \Psi_{D}(w, y)\right|$, hence exponentially small.

However, because $s^{*}(y) \approx y / 2$ for large $y$, upon increasing $y$ the saddle point $s^{*}(y)$ eventually exits the integration interval $0<s<w$ (i.e. for $y \gtrsim 2 w$ we have $s^{*}(y)>w$ ). Obviously, the saddle-point expansion becomes inaccurate for such a large $y$. Under such circumstances, the integrand in equation (A.18) is maximal at $s=w$, which gives the large- $y$ expansion

$$
F_{D}^{(\text {Extreme })}(y, w) \sim \frac{\mathrm{e}^{\Psi_{D}(y, w)}}{\left|\partial_{\mathrm{w}} \Psi_{D}(w, y)\right|}=\frac{\exp \left[-w-\frac{y^{2}}{4 w}\right]}{w^{D / 2}} \frac{4 w^{2}}{y^{2}-2 D w-4 w^{2}} .
$$

In summary, for any (dimensionless) time variable $w$ one may identify a (dimensionless) crossover length $l_{\mathrm{co}}$ through $s^{*}\left(l_{\mathrm{co}}\right)=w$. If $y \ll l_{\text {co }}$ then $F_{D}(y, w)$ is given to an excellent accuracy by its equilibrium limit, equation (A.4). Instead, for $y \gg l_{\text {co }}$ the asymptotic behavior is given by equation (A.22). Equation (A.20) provides asymptotic estimates for the crossover length,

$$
l_{\mathrm{co}}(w \rightarrow \infty) \sim 2 w, \quad \text { and } \quad l_{\mathrm{co}}(w \rightarrow 0) \sim \sqrt{2 D w} .
$$

\section{A.3. Back to real space: the computation of $\boldsymbol{F}_{D}(y, z)$}

For the sake of completeness, let us sketch the derivation of equation (A.3). We need to perform the inverse Fourier transform:

$$
G\left(\boldsymbol{r}, t_{\mathrm{w}}\right)=\int \frac{\mathrm{d}^{D} \boldsymbol{p}}{(2 \pi)^{D}} \mathrm{e}^{\mathrm{i} \boldsymbol{p} \cdot \boldsymbol{r}} \frac{\left(1-\exp \left[-2 t_{\mathrm{w}}\left(p^{2}+\xi_{\exp }^{-2}\right)\right]\right)}{p^{2}+\xi_{\exp }^{-2}} .
$$

After introducing the (dimensionless) length and time variables $y$ and $w$, recall equation (A.2), as well as the dimensionless momentum $\boldsymbol{u} \equiv \boldsymbol{p} \xi_{\exp }$, we find

$$
G\left(\boldsymbol{r}, t_{\mathrm{w}}\right)=\xi_{\exp }^{2-D} F_{D}(y, w), F_{D}(y, w)=\int \frac{\mathrm{d}^{D} \boldsymbol{u}}{(2 \pi)^{D}} \mathrm{e}^{\mathrm{i} \boldsymbol{u} \cdot \boldsymbol{y}} \frac{1-\mathrm{e}^{-w\left(u^{2}+1\right)}}{u^{2}+1} .
$$

Next, we note that the derivative with respect to $w$ of $F_{D}(y, w)$ can be computed by derivating under the integral sign (we are left with a Gaussian integral):

$$
\partial_{\mathrm{w}} F_{D}(y, w)=\int \frac{\mathrm{d}^{D} \boldsymbol{u}}{(2 \pi)^{D}} \mathrm{e}^{\mathrm{i} \boldsymbol{u} \cdot \boldsymbol{y}} \mathrm{e}^{-w\left(u^{2}+1\right)}=\frac{1}{(4 \pi)^{D / 2}} \frac{\exp \left[-w-\frac{y^{2}}{4 w}\right]}{w^{D / 2}} .
$$

Finally, because $F_{D}(y, w=0)=0$, equation (A.3) is recovered from

$$
F_{D}(y, w)=F_{D}(y, w)-F_{D}(y, w=0)=\int_{0}^{w} \mathrm{~d} s \partial_{s} F_{D}(y, s) .
$$


Out-of-equilibrium 2D Ising spin glass: almost, but not quite, a free-field theory

\section{References}

[1] Ballesteros H G, Cruz A, Fernandez L A, Martín-Mayor V, Pech J, Ruiz-Lorenzo J J, Tarancon A, Tellez P, Ullod C L and Ungil C 2000 Phys. Rev. B 62 14237-45

[2] Palassini M and Caracciolo S 1999 Phys. Rev. Lett. 82 5128-31

[3] Fisher D S and Huse D A 1988 Phys. Rev. B 38 373-85

[4] Rieger H, Steckemetz B and Schreckenberg M 1994 Europhys. Lett. 27485

[5] Marinari E, Parisi G, Ruiz-Lorenzo J and Ritort F 1996 Phys. Rev. Lett. 76 843-6

[6] Kisker J, Santen L, Schreckenberg M and Rieger H 1996 Phys. Rev. B 53 6418-28

[7] Joh Y G, Orbach R, Wood G G, Hammann J and Vincent E 1999 Phys. Rev. Lett. 82 438-41

[8] Berthier L and Bouchaud J P 2002 Phys. Rev. B 66054404

[9] Jönsson P E, Yoshino H, Nordblad P, Aruga Katori H and Ito A 2002 Phys. Rev. Lett. 88257204

[10] Berthier L and Young A P 2004 Phys. Rev. B 69184423

[11] Jiménez S, Martín-Mayor V and Pérez-Gaviro S 2005 Phys. Rev. B 72054417

[12] Berthier L and Young A P 2005 Phys. Rev. B 71214429

[13] Jaubert L C, Chamon C, Cugliandolo L F and Picco M 2007 J. Stat. Mech. P05001

[14] Belletti F et al 2008 Phys. Rev. Lett. 101157201

[15] Aron C, Chamon C, Cugliandolo L F and Picco M 2008 J. Stat. Mech. P05016

[16] Belletti F et al 2009 J. Stat. Phys. 1351121

[17] Guchhait S and Orbach R 2014 Phys. Rev. Lett. 112126401

[18] Manssen M and Hartmann A K 2015 Phys. Rev. B 91174433

[19] Baity-Jesi M et al 2017 Proc. Natl Acad. Sci. 114 1838-43

[20] Baity-Jesi M et al 2017 Phys. Rev. Lett. 118157202

[21] Guchhait S and Orbach R L 2017 Phys. Rev. Lett. 118157203

[22] Fernández L A, Marinari E, Martín-Mayor V, Parisi G and Ruiz-Lorenzo J 2018 An experiment-oriented analysis of 2d spin-glass dynamics: a twelve time-decades scaling study (arXiv:1805.06738)

[23] Boettcher S 2005 Phys. Rev. Lett. 95197205

[24] Guchhait S, Kenning G G, Orbach R L and Rodriguez G F 2015 Phys. Rev. B 91014434

[25] Guchhait S and Orbach R L 2015 Phys. Rev. B 92214418

[26] Zhai Q, Harrison D C, Tennant D, Dalhberg E D, Kenning G G and Orbach R L 2017 Phys. Rev. B 95054304

[27] Parisi G 1988 Statistical Field Theory (Reading, MA: Addison-Wesley)

[28] Amit D J and Martín-Mayor V 2005 Field Theory, the Renormalization Group and Critical Phenomena 3rd edn (Singapore: World Scientific)

[29] Fernandez L A, Marinari E, Martin-Mayor V, Parisi G and Ruiz-Lorenzo J J 2016 Phys. Rev. B 94024402

[30] Jörg T, Lukic J, Marinari E and Martin O C 2006 Phys. Rev. Lett. 96237205

[31] Edwards S F and Anderson P W 1975 J. Phys. F: Met. Phys. 5965

[32] Edwards S F and Anderson P W 1976 J. Phys. F: Met. Phys. 61927

[33] Parisi G 1994 Field Theory, Disorder and Simulations (Singapore: World Scientific)

[34] Mydosh J A 1993 Spin Glasses: an Experimental Introduction (London: Taylor and Francis)

[35] Belletti F et al 2008 Comput. Phys. Commun. 178 208-16

[36] Khoshbakht H and Weigel M 2017 Domain-wall excitations in the two-dimensional Ising spin glass Phys. Rev. B 97064410

[37] Katzgraber H W, Lee L W and Young A P 2004 Phys. Rev. B 70014417

[38] Cooper F, Freedman B and Preston D 1982 Nucl. Phys. B 210210

[39] Caracciolo S, Edwards R G, Pelissetto A and Sokal A D 1993 Nucl. Phys. B 403 475-541

[40] Olver F W J, Lozier D W, Boisvert R and Clark C (ed) 2010 NIST Handbook of Mathematical Functions (Cambridge: Cambridge University Press)

[41] Yllanes D 2011 Rugged free-energy landscapes in disordered spin systems PhD Thesis Universidad Complutense de Madrid 